\title{
Modulation in voluntary neural drive in relation to muscle soreness
}

\author{
S. Racinais $\cdot$ A. Bringard $\cdot$ K. Puchaux $\cdot$ T. D. Noakes $\cdot$ \\ S. Perrey
}

Accepted: 22 October 2007 / Published online: 3 November 2007

(C) The Author(s) 2007

\begin{abstract}
The aim of this study was to investigate whether (1) spinal modulation would change after nonexhausting eccentric exercise of the plantar flexor muscles that produced muscle soreness and (2) central modulation of the motor command would be linked to the development of muscle soreness. Ten healthy subjects volunteered to perform a single bout of backward downhill walking exercise (duration $30 \mathrm{~min}$, velocity $1 \mathrm{~ms}^{-1}$, negative grade $-25 \%$, load $12 \%$ of body weight). Neuromuscular test sessions [H-reflex, M-wave, maximal voluntary torque (MVT)] were performed before, immediately after, as well as 1-3 days after the exercise bout. Immediately after exercise there was a $-15 \%$ decrease in MVT of the plantar flexors partly attributable to an alteration in contractile properties $(-23 \%$ in electrically evoked mechanical twitch). However, MVT failed to recover before the third day whereas the contractile properties had significantly recovered within the first day. This delayed recovery of MVT was likely related to a decrement in voluntary muscle drive. The decrease in voluntary activation occurred in the absence of any variation in spinal modulation estimated from the H-reflex. Our findings suggest the development of
\end{abstract}

S. Racinais $\cdot$ A. Bringard $\cdot$ K. Puchaux $\cdot$ S. Perrey

Motor Efficiency and Deficiency Laboratory,

EA 2991, UFR STAPS, Montpellier, France

S. Racinais $(\square)$

Exercise and Sports Science Department, ASPETAR, Qatar Orthopaedic and Sports Medicine Hospital,

PO Box 29222 Doha, Qatar

e-mail: contact@sebastienracinais.com

T. D. Noakes

MRC/UCT Research Unit for Exercise Science

and Sports Medicine, Sports Science Institute of SA,

Cape Town, South Africa a supraspinal modulation perhaps linked to the presence of muscle soreness.

Keywords Exercise $\cdot$ Neuromuscular $\cdot$ Central Fatigue . Eccentric · DOMS

\section{Introduction}

In an exercise inducing muscle damage, inadequate neural drive can be an attempt of the neuromuscular system to protect the muscle-tendon unit from additional damage (Strojnik and Komi 2000; Nicol et al. 2006). This inadequate neural drive could be the result from a combination of three factors: the conscious and unconscious will of the subject to reduce the exercise intensity; an inability of the motor cortex to generate sufficient output to maximally activate the muscle; and/or a decreased transmission of the supraspinal input to the muscle by the spinal motor axons.

A decrement in H-reflex amplitude has been observed immediately after an exhausting voluntary contraction of a single muscle group (i.e., Duchateau et al. 2002; Garland and McComas 1990; Kuchinad et al. 2004). It has been proposed that this decline in the transmission of the action potentials from the Ia afferent to the $\alpha$-motoneuron may be a consequence of a presynaptic inhibition mediated by group III and IV afferents (Bigland-Ritchie et al. 1986; Duchateau et al. 2002; Garland and McComas 1990; Garland 1991; Woods et al. 1987) induced by muscle damage (Avela et al. 2006). Furthermore, increased group III and IV muscle afferent inputs could induce $\mathrm{H}$-reflex depression when muscle soreness progresses as muscle pain is believed to reflect activity in group III and IV muscle afferents (O'Connor and Cook 1999). But only a few studies have observed a decrease in H-reflex amplitude after 
exercise of multiple muscle groups as occurs for example in running (Avela et al. 1999; Bulbulian and Bowles 1992; Racinais et al. 2007b) raising the question of whether spinal modulation occurs after whole body exercise. Recently it has been shown that walking backward induces muscle soreness in the muscles of the lower limb (Nottle and Nosaka 2005). Accordingly, this exercise model allows the study of the effects of muscle soreness on alteration in neural drive. Thus the goal of this study was to determine whether the impaired exercise performance of muscles with delayed onset muscle soreness (DOMS) is due to an alteration in neural drive related to spinal modulation.

\section{Methods}

\section{Subjects}

Ten healthy subjects (eight males and two females; age $27 \pm 1$ years; weight $68 \pm 2 \mathrm{~kg}$; height $174 \pm 2 \mathrm{~cm}$; data in mean \pm SEM) gave informed, written consent to participate in this study. The procedures complied with the Helsinki declaration for human experimentation and were approved by the local Ethics Committee. None of the subjects suffered from muscle soreness or ankle injuries. Subjects were asked to avoid caffeine intake within the 8-h preceding the test and to avoid all vigorous activity during the 24-h preceding the test. Subjects were also asked to refrain from analgesic intake all along the protocol, which could have disturbed DOMS perception.

\section{Experimental procedures}

Subjects visited our laboratory on four consecutive days (Fig. 1). The first day consisted of a neuromuscular test session (described subsequently) followed by a backward walking exercise (description below) followed by further neuromuscular testing. On the second, third and fourth days, subjects returned to the laboratory at the same hour of day that they had finished the walking exercise and performed the neuromuscular testing.

\section{Neuromuscular tests}

The neuromuscular tests are described in Fig. 1. All the neuromuscular tests began with the determination of the stimulation intensity required to elicit a maximal $\mathrm{H}$-reflex $\left(H_{\max }\right)$. Afterwards, three $H_{\max }$ (interspaced by $20 \mathrm{~s}$ ) and three maximal $M$-wave amplitudes $\left(M_{\max }\right)$ interspaced by $8 \mathrm{~s}$ were elicited from the relaxed muscle. The amplitude of the three twitches evoked at $H_{\max }$ and $M_{\max }$ intensities were averaged for subsequent analysis in both the soleus and gastrocnemius medialis. Thereafter, subjects were instructed

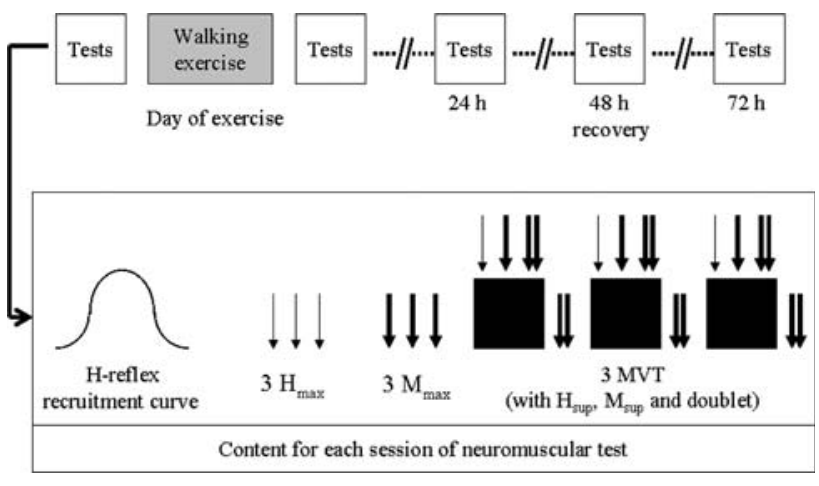

Fig. 1 Experimental design. Thin arrow indicates stimulation at $H_{\max }$ intensity, simple thick arrow indicates stimulation at $M_{\max }$ intensity, double thick arrow indicates doublet at $M_{\max }$ intensity

to perform three maximal voluntary torque (MVT) contractions of the plantar flexor muscles, each for $5 \mathrm{~s}$. Subjects were verbally encouraged to perform maximally. A superimposed stimulus ( $H_{\max }$ intensity) was evoked during each MVT plateau to obtain the H-reflex during contraction $\left(H_{\text {sup }}\right)$. Then, another superimposed stimulus $\left(M_{\max }\right.$ intensity) was evoked in order to obtain a superimposed M-wave $\left(M_{\text {sup }}\right)$ during voluntary contraction. Finally, a doublet (two electrically evoked twitches, $10 \mathrm{~ms}$ apart, $M_{\max }$ intensity) was evoked during each plateau (superimposed twitch) and another doublet was evoked $4 \mathrm{~s}$ after each MVT (potentiated twitch). The ratio of the amplitude of the superimposed twitch torque over the amplitude of a twitch evoked at rest $4 \mathrm{~s}$ after the MVT was used to assess the level of voluntary activation (VA) (Allen et al. 1995). According to the twitch interpolation method (Allen et al. 1995), the percentage of VA was calculated as follow: VA $(\%)=(1-$ Superimposed Twitch/Potentiated Twitch) $\times 100$.

\section{Muscle soreness assessment}

A subjective evaluation of the extent of DOMS in the plantar flexor muscles was performed before each neuromuscular test by completing two subjective scales for evaluation of DOMS. The first was a visual scale of $9 \mathrm{~cm}$ without any graduation (horizontal line ranging from no pain at the left to extreme pain at the right). The second was a Lickert scale with seven items (from 0: no pain to 6: severe pain limiting movement, Vickers 2001).

\section{Backward downhill walking exercise}

Subjects exercised by walking on a motorized treadmill (S2500, HEF Techmachine, France) for 30-min at a constant velocity of $1 \mathrm{~ms}^{-1}$ with a negative grade of $-25 \%$. To increase the eccentric loading on the plantar flexor muscles, 
the walk was performed backward (Nottle and Nosaka 2005) whilst wearing a vest loaded with an additional weight equivalent to $12 \%$ of body weight.

Measurement and calculations

\section{Torque measurement}

The MVT of the plantar flexor muscles was recorded by a dynamometric pedal (Captels, St Mathieu de Treviers, France). Subject position was standardized with hip, knee and ankle angulations of $90^{\circ}$, and foot securely strapped on the pedal.

\section{Evoked potentials}

The tibial nerve was stimulated with a cathode electrode with a diameter of $9 \mathrm{~mm}$ placed in the popliteal cavity (Contrôle Graphique Medical, Brie-Comte-Robert, France). Subjects were in a standardized position with motionless head (Zehr 2002) and a standardized environment (i.e., same time-of-day, silent room, constant lighting). Furthermore, a constant pressure was applied to the electrode with the use of a strap. This was controlled by an air pressure-recorder (Kikuhime, TT MediTrade, Soro, Denmark) located under the strap. The anode $(10 \mathrm{~cm} \times$ $5 \mathrm{~cm}$, Medicompex, Ecublens, Switzerland) was positioned distal to the patella. Electrical stimulations (400 V, rectangular pulse of $0.2 \mathrm{~ms}$ ) were delivered by a high-voltage stimulator (Digitimer DS7AH, Digitimer, Hertfordshire, England). The amperage was adjusted for each subject during the familiarization session. During this first session, the amperage was increased progressively $(10 \mathrm{~mA}$ increment) until a plateau in twitch mechanical response [peak twitch (Pt)] and $M_{\max }$ were observed. With increasing stimulation intensity, the H-reflex response initially increased progressively before decreasing and then disappearing. Thereafter, the intensity needed to obtain $H_{\max }$ was adjusted by $1 \mathrm{~mA}$. The stimulation intensity needed to obtain $H_{\max }$ was determined before each test session but with a simplified procedure based on the knowledge of the intensity used during the first test session. This adjustment seemed necessary for the H-reflex in view of the important variation occurring in $H_{\max }$ for a small variation in stimulation conditions (e.g., intensity, localization of the cathode).

\section{Recordings}

Evoked Pt torque was recorded in relaxed muscle by the same ergometer than MVT. Both MVT and Pt were measured with the knee at $90^{\circ}$ to reflect the changes occurring in the soleus. Reflex waves for both the soleus, which provides the highest responses due to the activation of the slow twitch fibre by H-reflex (Buchthal and Schmalbruch 1970), and the gastrocnemius medialis, which is particularly susceptible to be affected by the walking exercise were recorded with $9 \mathrm{~mm}$ diameter bipolar $\mathrm{Ag} / \mathrm{AgCl}$ electrodes (Contrôle Graphique Medical, Brie-Comte-Robert, France) with an inter-electrode distance of $25 \mathrm{~mm}$. The reference electrode was placed on the wrist. Low impedance between the two electrodes $(<5 \mathrm{k} \Omega)$ was obtained by abrading and washing the skin with emery paper and cleaning with alcohol. Signals were amplified and filtered (band pass 30 $500 \mathrm{~Hz}$, gain $=1,000$ ), and recorded at high frequency $(2,000 \mathrm{~Hz})$. The compound muscle action potentials were recorded using MP30 hardware (Biopac Systems Inc., Santa Barbara, CA, USA) and dedicated software (BSL Pro Version 3.6.7, Biopac Systems Inc., Santa Barbara, CA, USA). The same equipment was also used to drive the stimulator.

\section{Calculation}

The Pt may be considered as an index of the contractile properties and $M_{\max }$ amplitude represents an index of sarcolemmal excitability. Because no evidence exists to show that data recorded at rest reflects the responses of the neuromuscular system during contraction, $H_{\text {sup }}$ amplitude was recorded during MVT to complement $H_{\max }$ amplitude at rest. To ensure that any changes in the evoked $H_{\max }$ and $H_{\text {sup }}$ amplitudes were not due to changes at the muscle fiber membrane or neuromuscular junction (Cupido et al. 1996), we normalized these recordings to the M-wave amplitude recorded under the same testing conditions, that is the $H_{\text {max }} / M_{\text {max }}$ and $H_{\text {sup }} / M_{\text {sup }}$ ratios. These ratios may be considered as a global index of the spinal modulation produced by presynaptic inhibition, motoneuron excitability, collision in antidromically activated axons and Renshaw cell inhibition, acting individually or in concert.

\section{Statistical analysis}

Each variable was tested for normality using the Skewness and Kurtosis tests with acceptable $Z$ values not exceeding \pm 1.5 . With the assumption of normality confirmed, parametric tests could be performed. The effect of the walking exercise was analyzed for each variable by a one-way analysis of variance for repeated measures (five test sessions). The contrast method was applied as post hoc to further investigate the effect of both the exercise and the recovery. Because VA level failed to display a normal distribution, a Friedman test was used instead of the ANOVA. Statistical analyses were performed with Systat software (Systat, Evanston, IL, USA). Data are reported as mean \pm SEM and the level of statistical significance was set at $P<0.05$. 


\section{Results}

Maximal voluntary torque, voluntary activation and contractile properties

The MVT significantly changed across the 4 days following the walking test $\left(F_{4,36}=3.8, P<0.02\right.$, Fig. $\left.2 \mathrm{a}\right)$. Post hoc analysis showed a significant decrease in MVT after the walking exercise and which persisted during the next two days $\left(F_{1,9}=12.33, P<0.01\right)$. A significant recovery in MVT was observed on the third day (48-h versus $72-\mathrm{h}$ after: $F_{1,9}=9.64, P<0.02$ ).

In line with the evolution observed in MVT, post hoc analysis showed a significant decrease in VA level after the walking exercise (pre versus post-exercise: $P<0.02$, Fig. 2b) which failed to recover by 48-h (post-exercise versus 24-h and 48-h after exercise, NS). However there was a significant recovery by 72 -h (post-exercise versus after $72-\mathrm{h}$, $P<0.005)$.

The electrically evoked Pt also displayed a significant variation following walking exercise $\left(F_{4,36}=14.07\right.$, $P<0.001$, Fig. 2c). Post hoc analysis revealed a significant decrease in $\mathrm{Pt}$ after the exercise $\left(F_{1,9}=58.34, P<0.001\right)$ followed by a significant recovery thereafter $\left(F_{1,9}=24.76\right.$, $P<0.001)$.

\section{Subjective DOMS}

The subjects feeling of DOMS increased significantly in the days following the walking exercise $\left(F_{4,27}>28, P<0.001\right.$ for both scales used, Fig. 2d). Post hoc analysis displayed significantly higher subjective DOMS for the 3 days following exercise compared to the termination of exercise
$\left(F_{1,9}>35, P<0.001\right.$, for both scales). Muscle soreness reached a maximum 48-h after exercise and began to recover by 72 -h (48-h versus 72 -h after exercise: $F_{1,9}>12$, $P<0.01$ for both scales).

\section{Evoked potentials}

An example of evoked potentials recorded in a representative subject is displayed in Fig. 3 and the mean values are displayed in Table 1. The walking exercise failed to induce significant changes in the evoked potentials both at rest ( $M_{\max }, F_{4,36}=1.60$ and 0.60 for soleus and gastrocnemius medialis respectively, NS) and during the voluntary contraction ( $M_{\text {sup }}, F_{4,36}=0.46$ and 2.20 for soleus and gastroc nemius medialis respectively, NS). Furthermore, the reflex waves calculated both at rest $\left(H_{\max } / M_{\max }\right.$ ratio $)$ and during contraction ( $H_{\text {sup }} / M_{\text {sup }}$ ratio) also did not change significantly (all $F_{4,36}<0.98$, NS, Table 1 ).

\section{Discussion}

The downhill walking exercise induced a significant decrease in the MVT of the plantar flexor muscles. Immediately after the walking exercise, the torque decrement of $-15 \%$ (Fig. 2a) appeared to be caused partly by an alteration in muscle contractile properties (i.e., $-23 \%$ for $\mathrm{Pt}$, Fig. 2c). This alteration is typically referred to as "peripheral fatigue" [for a review, see Millet and Lepers 2004]. Furthermore, this peripheral fatigue was also associated with a decrease in VA (i.e., -5.3\%, Fig. 2b) suggesting the concomitant existence of a "central modulation" [for a review, see Gandevia 2001]. The first finding of this study
Fig. 2 Evolution of voluntary torque (a), voluntary activation (b), electrically evoked peak twitch (c) and subjective delayed onset muscle soreness (d, black rectangle: analogic visual scale, white rectangle: Lickert scale) across the experimental sessions. Data in mean \pm SEM, Asteriskindicates value or group of values significantly different from the other values of the graph $(P<0.05)$
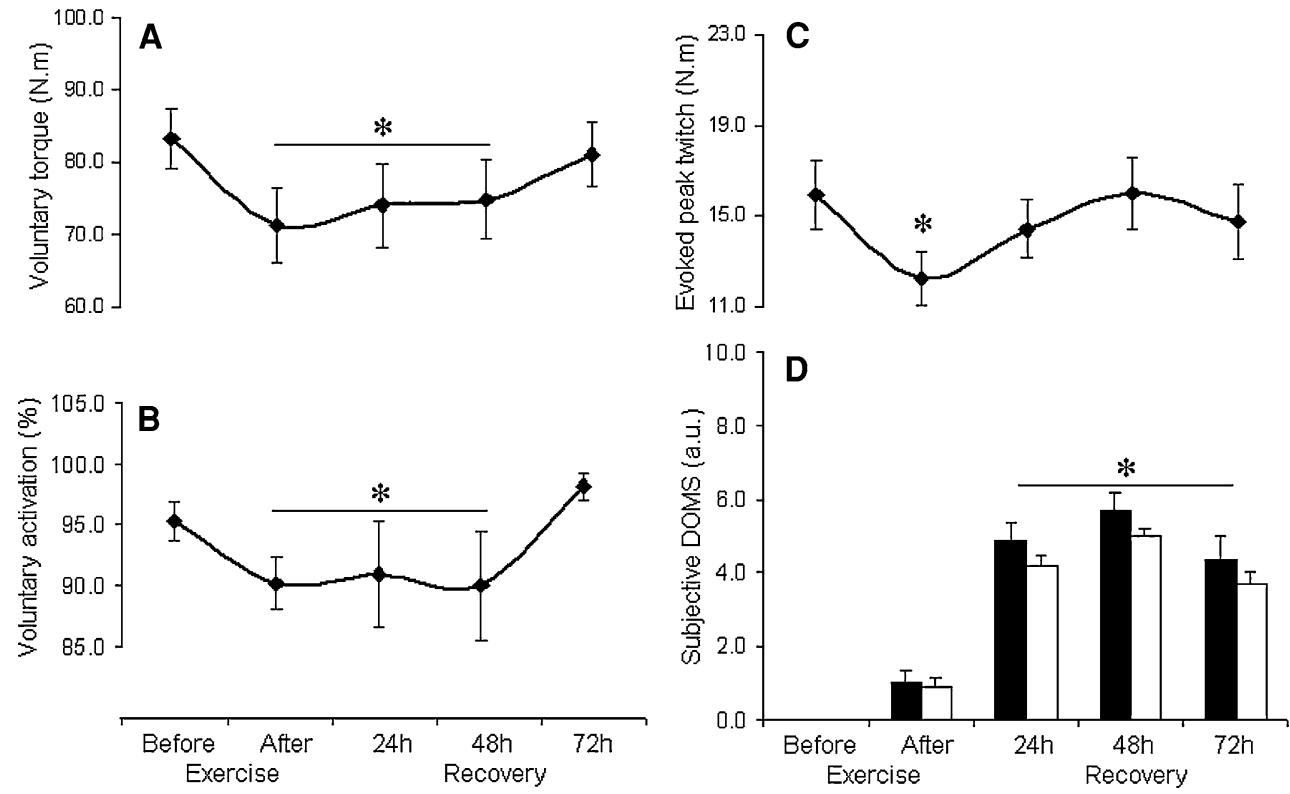
Fig. 3 Example of evoked potentials recorded in a representative subject. Each drawing represents the average of three recordings obtained on a relaxed muscle

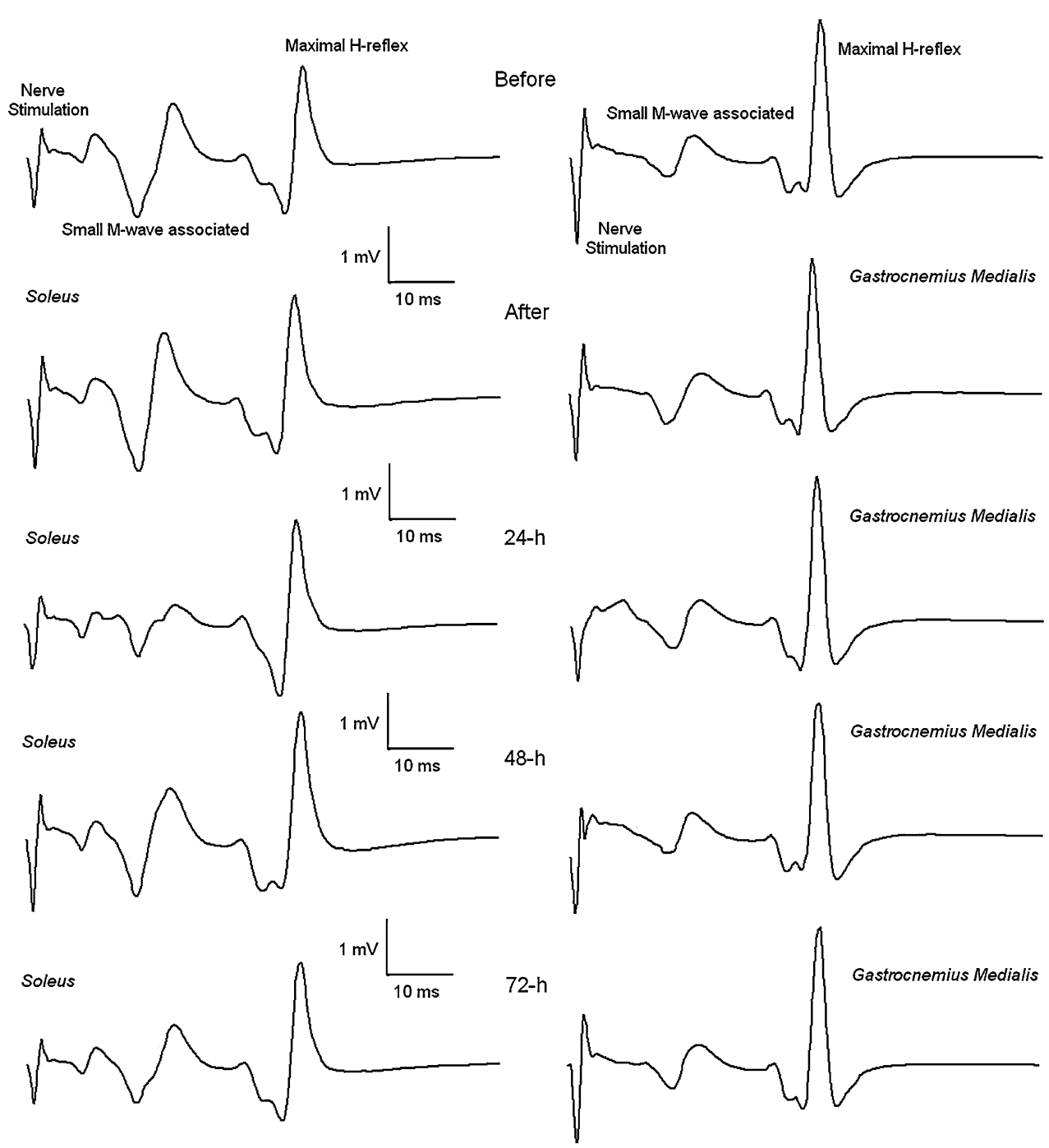

is that the maximum voluntary torque failed to recover before the third day (i.e., $-12 \%$ and $-10 \%$ after $24-\mathrm{h}$ and 48-h of recovery, respectively, Fig. 2a) whereas the measure of the (peripheral) contractile properties had recovered significantly within the first $24 \mathrm{~h}$ after exercise $(P<0.01$, Fig. 2c). This delayed recovery in MVT appeared to be mainly associated with a decrease in voluntary muscle activation (Fig. 2b). The time course of change in VA presents similarities with the time course of torque changes.

A significant decrease in the VA reaching the muscle has previously been observed after prolonged (Millet et al. 2002, 2003) and short-duration (Racinais et al. 2007a) fatiguing exercise, but our data showed that a backward downhill walking exercise did produce a decrease in muscle activation persisting for a few days after the exercise. It has been suggested that this central component could explain as well the reduction in force production by the respiratory muscles after heavy exercise (Verin et al. 2004) and represents a protective mechanism in order that peripheral muscle fatigue does not exceed a critical threshold (Amann et al.
2006). This central protection of the muscle from further peripheral fatigue and damage will be performed at the expense of a truly maximal performance in which all the motor units are activated (Gandevia et al. 1996).

It has recently been suggested that a "central governor" in the brain regulates the extent of skeletal muscle recruitment during exercise (Noakes et al. 2005). According to this theory, the sensation of fatigue is the conscious interpretation of these homoeostatic control mechanisms during prolonged exercise (Noakes et al. 2005). Our results could partly support this theory but with the proviso that the modulation of central activation in this experiment appears to be related not only to fatigue immediately after exercise but also to the development of DOMS the days following the exercise. However, regulation of muscle recruitment by the brain (i.e., supraspinal regulation) is not the only system that could explain the observed decrease in skeletal muscle activation since reflex pathways need also to be considered (i.e., spinal modulation). Previous results showed impairment in VA after eccentric exercise when VA was estimated by 
Table 1 Evolution of maximal compound action potential electrically evoked at rest $\left(M_{\max }\right)$ and during MVT $\left(M_{\text {sup }}\right)$, normalized H-reflex at rest $\left(H_{\text {max }} / M_{\text {max }}\right)$ and during MVT $\left(H_{\text {sup }} / M_{\text {sup }}\right)$

\begin{tabular}{|c|c|c|c|c|c|c|}
\hline & \multicolumn{2}{|c|}{ Exercise } & \multicolumn{3}{|c|}{ Recovery } & \multirow{2}{*}{$\begin{array}{l}\text { Statistical } \\
\text { analysis }\end{array}$} \\
\hline & Before & After & 24-h & 48-h & 72-h & \\
\hline \multicolumn{7}{|l|}{ Soleus } \\
\hline \multirow[t]{2}{*}{$M_{\max }(\mathrm{mV})$} & 7.35 & 6.80 & 7.83 & 7.74 & 7.18 & NS \\
\hline & 0.53 & 0.49 & 0.8 & 0.58 & 0.75 & \\
\hline \multirow[t]{2}{*}{$M_{\text {sup }}(\mathrm{mV})$} & 8.87 & 8.13 & 9.05 & 8.91 & 8.75 & NS \\
\hline & 1.14 & 0.76 & 1.13 & 0.97 & 1.29 & \\
\hline \multirow[t]{2}{*}{$H_{\max } / M_{\max }$ ratio } & 0.36 & 0.37 & 0.38 & 0.37 & 0.36 & NS \\
\hline & 0.10 & 0.09 & 0.10 & 0.10 & 0.09 & \\
\hline \multirow[t]{2}{*}{$H_{\text {sup }} / M_{\text {sup }}$ ratio } & 0.39 & 0.45 & 0.39 & 0.34 & 0.34 & NS \\
\hline & 0.10 & 0.10 & 0.08 & 0.08 & 0.06 & \\
\hline \multicolumn{7}{|c|}{ Gastrocnemius medialis } \\
\hline \multirow[t]{2}{*}{$M_{\max }(\mathrm{mV})$} & 6.61 & 6.10 & 6.42 & 6.83 & 6.50 & NS \\
\hline & 1.07 & 0.76 & 0.64 & 1.0 & 0.92 & \\
\hline \multirow[t]{2}{*}{$M_{\text {sup }}(\mathrm{mV})$} & 9.40 & 7.04 & 8.24 & 7.95 & 8.14 & NS \\
\hline & 1.29 & 1.06 & 0.81 & 0.89 & 2.26 & \\
\hline \multirow[t]{2}{*}{$H_{\max } / M_{\max }$ ratio } & 0.18 & 0.19 & 0.22 & 0.19 & 0.19 & NS \\
\hline & 0.05 & 0.05 & 0.05 & 0.05 & 0.05 & \\
\hline
\end{tabular}

Data in mean \pm SEM

nerve stimulation but not by cortical stimulation (Prasartwuth et al. 2005). That suggests that the VA deficit lies within these two site of stimulations, i.e., in the motor cortex or at spinal level (Prasartwuth et al. 2005), rather than a governor upstream to the motor cortex.

In the present study, we used H-reflex amplitude as a tool to evaluate the spinal modulation (Aagaard et al. 2002; Schieppati 1987) to provide a differentiation within these possible regulatory mechanisms. The DOMS produced by our experimental protocol would have induced an increased discharge of group III and IV muscle afferents (Avela et al. 1999) and thus a pre-synaptic inhibition of the transmission from the Ia afferent stimulation to the $\alpha$-motoneurons (Avela et al. 2006; Bigland-Ritchie et al. 1986; Duchateau et al. 2002; Garland and McComas 1990; Garland 1991; Woods et al. 1987). The extent to which this inhibition occurs will depend on whether the input producing the inhibition is ongoing or has ceased. But, in theory, increased group III and IV muscle afferent input induced by the DOMS should induce H-reflex depression as the soreness progresses. Indeed, muscle pain is believed to reflect activity in group III and IV muscle afferents (O'Connor and Cook 1999). However, our results failed to show significant variations in the evoked reflex-wave amplitudes throughout the experiment (i.e., $H_{\max } / M_{\max }$ and $H_{\text {sup }} / M_{\text {sup }}$ ratios) suggesting that the motoneuron pool excitability was well preserved.

A number of previous studies have demonstrated a significant H-reflex decrease in the exercised muscle group after an exhausting voluntary contraction of that muscle group (i.e., Duchateau et al. 2002; Garland and McComas 1990; Kuchinad et al. 2004). But only a few studies have observed this decrease after more generalized muscular exercise such as running, (Avela et al. 1999; Bulbulian and Bowles 1992; Racinais et al. 2007b). Our results support these findings by showing that after a 30-min downhill walking exercise, electrically evoked reflex-wave activity was not decreased. Thus we conclude that non-exhausting walking exercise, sufficient to induce significant DOMS seems not to induce alteration of the spinal modulation.

Since the persistence of a decrease in VA during several days in this study could not be explained by spinal modulation, it seems likely that a supraspinal component must have played a part. Accordingly the changes in VA that occurred at the same time that the subjective symptoms of DOMS suggest a supraspinal regulation of muscle recruitment. Indeed it has been known for some time that exercise performance is regulated at least in part by supraspinal factors. For example, Bigland-Ritchie (1981) showed that central fatigue during repeated isometric contraction is minimized by exhorting the subject to produce a "super" effort at the end of each voluntary contraction. Our results add to that interpretation by suggesting the possibility that supraspinal modulation can also occurs after locomotory activity such as walking, even without exhaustion.

As we have already argued, the observed increase in DOMS could represent the conscious interpretation of an increased discharge of group III and IV muscle afferents (O'Connor and Cook 1999). Even though we failed to observe a significant alteration of the spinal modulation at the time of increased muscle soreness, this would not prove that the discharge of these afferents had not increased since it is still unclear whether group III and IV muscle afferents induce a post-exercise decrease in motoneurons excitability in healthy humans. Indeed, previous studies have shown that maintained firing of ischaemically sensitive group III and IV muscle afferents does not influence the altered muscle responses to cortical or corticospinal stimulation observed after fatiguing exercise (Andersen et al. 2003; Gandevia et al. 1996; Taylor et al. 2000). All these findings might suggest that, after exercise, increased output from group III and IV muscle afferents may not directly inhibit the motoneurons but may act upstream of the motor cortex to impair voluntary descending motor drive (Taylor et al. 2006).

Accordingly, a novel contribution of this study is that VA significantly recovered on the third day, when the DOMS displayed also a significant decrement $\left(F_{1,9}>12\right.$, $P<0.01$, for the both scales). Although this temporal relationship does not prove causality, this finding could suggest a relationship between the persistence of the decrease in VA and the subjective symptoms of DOMS in these subjects. This observation is consistent with the data of Le Pera 
et al. (2001) showing that muscle pain could induce a longlasting depression in motor activation. Their data suggest that this inhibition in motor system excitability could be linked to a decreased excitability of the motor cortex as well as spinal modulation (Le Pera et al. 2001). However, Prasartwuth et al. (2005) observed a different time course in muscle soreness after eccentric exercise and changes in VA leading these authors to suggest that muscle pain did not directly cause the change in voluntary drive.

These different data emphasised the complexity of the relation within muscle soreness and voluntary muscle drive. It has been recently observed that motoneuron excitability in elbow flexors, but not extensors, was able to recover when ischemia is maintained after fatiguing contractions (Martin et al. 2006), a finding that suggests differential influences of group III and IV muscle afferents on different motoneuron pools (Martin et al. 2006). In this study concerning the plantar flexors, we observed some statistical similarities within DOMS and VA (i.e., lowest value of VA at 48-h when DOMS was the highest and significant recovery for the both at 72-h). However, from a functional point of view, VA returned at control level at 72-h whereas DOMS at 72-h was not less than DOMS at 24-h, and VA decreased after exercise when DOMS was weak. That suggests that subjective DOMS of the plantar flexors can not be considered as an objective indicator of VA capability.

\section{Conclusion}

It was recently reported that a 90 min bout of flat running exercise produced a modification in spinal modulation (Racinais et al. 2007b). The present study showed that a 30-min downhill walking exercise failed to induce the same modification. However there was a significant decrease in VA during maximal voluntary contractions performed during the days after eccentric exercise that produced DOMS. This suggested the occurrence of a supraspinal modulation of muscle activation during this period when muscle contractile properties had fully recovered following the eccentric exercise. Furthermore, the persistence of the decrement for several days suggests that this modulation was not caused by an acute exercise-related physiological or biochemical alteration in the motor cortex, so-called central fatigue but more likely represents a modulation which may be partly linked to the muscle soreness.

Open Access This article is distributed under the terms of the Creative Commons Attribution Noncommercial License which permits any noncommercial use, distribution, and reproduction in any medium, provided the original author(s) and source are credited.

\section{References}

Aagaard P, Simonsen EB, Andersen JL, Magnusson P, Dyhre-Poulsen P (2002) Neural adaptation to resistance training: changes in evoked V-wave and H-reflex responses. J Appl Physiol 92:2309-2318

Allen GM, Gandevia SC, McKenzie DK (1995) Reliability of measurements of muscle strength and voluntary activation using twitch interpolation. Muscle Nerve 18:593-600

Amann M, Eldridge MW, Lovering AT, Stickland MK, Pegelow DF, Dempsey JA (2006) Arterial oxygenation influences central motor output and exercise performance via effects on peripheral locomotor muscle fatigue in humans. J Physiol 575:937-952

Andersen B, Westlund B, Krarup C (2003) Failure of activation of spinal motoneurones after muscle fatigue in healthy subjects studied by transcranial magnetic stimulation. J Physiol 551:345-356

Avela J, Kyröläinen H, Komi PV, Rama D (1999) Reduced reflex sensitivity persists several days after long-lasting stretch-shortening cycle exercise. J Appl Physiol 86:1292-1300

Avela J, Finni J, Komi PV (2006) Excitability of the soleus reflex arc during intensive stretch-shortening cycle exercise in two powertrained athlete groups. Eur J Appl Physiol 97:486-493

Bigland-Ritchie B (1981) EMG and fatigue of human voluntary and stimulated contractions. Ciba Found Symp 82:130-156

Bigland-Ritchie B, Dawson NJ, Johansson RS, Lippold OCJ (1986) Reflex origin for the slowing of motoneurone firing rates in fatigue of human voluntary contractions. J Physiol 379:451-459

Buchthal F, Schmalbruch H (1970) Contraction times of twitches evoked by H-reflex. Acta Physiol Scand 80:378-382

Bulbulian R, Bowles DK (1992) Effect of downhill running on motoneuron pool excitability. J Appl Physiol 73:968-973

Cupido CM, Galea V, McComas AJ (1996) Potentiation and depression of the M wave in human biceps brachii. J Physiol 491:541550

Duchateau J, Balestra C, Carpentier A, Hainaut K (2002) Reflex regulation during sustained and intermittent submaximal contractions in humans. J Physiol 541:959-967

Gandevia SC (2001) Spinal and supraspinal factors in human muscle fatigue. Physiol Rev 81:1725-1789

Gandevia SC, Allen GM, Butler JE, Taylor JL (1996) Supraspinal factors in human muscle fatigue: evidence for suboptimal output from the motor cortex. J Physiol 490:529-536

Garland SJ (1991) Role of small diameter afferents in reflex inhibition during human muscle fatigue. J Physiol 435:547-558

Garland SJ, McComas AJ (1990) Reflex inhibition of human soleus muscle during fatigue. J Physiol 429:17-27

Kuchinad RA, Ivanova TD, Garland SJ (2004) Modulation of motor unit discharge rate and H-reflex amplitude during submaximal fatigue of the human soleus muscle. Exp Brain Res 158:345-355

Le Pera D, Graven-Nielsen T, Valeriani M, Oliviero A, Di Lazzaro V, Tonali PA, Arendt-Nielsen L (2001) Inhibition of motor system excitability at cortical and spinal level by tonic muscle pain. Clin Neurophysiol 112:1633-1641

Martin PG, Smith JL, Butler JE, Gandevia SC, Taylor JL (2006) Fatigue-sensitive afferents inhibit extensor but not flexor motoneurons in humans. J Neurosci 26:4796-4802

Millet GY, Lepers R (2004) Alterations of neuromuscular function after prolonged running, cycling and skiing exercises. Sports Med 34:105-116

Millet GY, Lepers R, Maffiuletti NA, Babault N, Martin V, Lattier G (2002) Alteration of neuromuscular function after an ultra-marathon. J Appl Physiol 92:486-492

Millet GY, Martin V, Lattier G, Ballay Y (2003) Mechanisms contributing to knee extensors strength loss after prolonged running exercise. J Appl Physiol 94:193-198 
Nicol C, Avela J, Komi PV (2006) The stretch-shortening cycle: a model to study naturally occurring neuromuscular fatigue. Sports Med 36:977-999

Noakes TD, St Clair Gibson A, Lambert EV (2005) From catastrophe to complexity: a novel model of integrative central neural regulation of effort and fatigue during exercise in humans: summary and conclusions. Br J Sports Med 39:120-124

Nottle C, Nosaka K (2005) The magnitude of muscle damage induced by downhill backward walking. J Sci Med Sport 8:264-273

O'Connor PJ, Cook DB (1999) Exercise and pain: the neurobiology, measurement, and laboratory study of pain in relation to exercise in humans. Exerc Sport Sci Rev 27:119-166

Prasartwuth O, Taylor JL, Gandevia SC (2005) Maximal force, voluntary activation and muscle soreness after eccentric damage to human elbow flexor muscles. J Physiol 567:337-348

Racinais S, Bishop D, Denis R, Lattier G, Mendez-Villaneuva A, Perrey S (2007a) Muscle de-oxygenation and neural drive to the muscle during repeated sprint cycling. Med Sci Sports Exerc 39:268-274

Racinais S, Girard O, Micallef JP, Perrey S (2007b) Failed excitability of spinal motoneurons induced by prolonged running exercise. J Neurophysiol 97:596-603

Schieppati M (1987) The Hoffman reflex: a means for assessing spinal reflex excitability and its descending control in man. Prog Neurobiol 28:345-376
Strojnik V, Komi PV (2000) Fatigue after submaximal intensive stretchshortening cycle exercise. Med Sci Sports Exerc 32:1314-1319

Taylor JL, Petersen N, Butler JE, Gandevia SC (2000) Ischaemia after exercise does not reduce responses of human motoneurones to cortical or corticospinal tract stimulation. J Physiol 525:793801

Taylor JL, Todd G, Gandevia SC (2006) Evidence for a supraspinal contribution to human muscle fatigue. Clin Exp Pharmacol Physiol 33:400-405

Verin E, Ross E, Demoule A, Hopkinson N, Nickol A, Fauroux B, Moxham J, Similowski T, Polkey MI (2004) Effects of exhaustive incremental treadmill exercise on diaphragm and quadriceps motor potentials evoked by transcranial magnetic stimulation. J Appl Physiol 96:253-259

Vickers AJ (2001) Time course of muscle soreness following different types of exercise. BMC Musculoskelet Disord 2:1471-1474

Woods JJ, Furbush F, Bigland-Ritchie B (1987) Evidence for a fatigueinduced reflex inhibition of motoneuron firing rates. J Neurophysiol 58:125-137

Zehr EP (2002) Considerations for use of the Hoffmann reflex in exercise studies. Eur J Appl Physiol 86:455-468 